\title{
CT and MR Imaging Findings of Sinonasal Schwannoma: A Review of 12 Cases
}

\author{
Y.S. Kim, H.-J. Kim, C.-H. Kim, and J. Kim
}

\begin{abstract}
SUMMARY: Schwannomas are benign tumors that are rarely found in the sinonasal cavity, and the purpose of this study was to characterize the CT and MR imaging findings of 12 patients with surgically proved sinonasal schwannomas. Assessed features include location, margin, shape, size, internal architecture, pattern and degree of enhancement, and associated bony wall changes. The characteristic CT and MR imaging findings of sinonasal schwannoma include a well-defined soft-tissue mass, most frequently occurring in the nasal cavity and ethmoid sinus with pressure remodeling of the adjacent bony wall. The tumors were isoattenuating on CT and predominantly isointense on both T1- and T2-weighted MR images, compared with the brain stem. Mild contrast enhancement on CT and strong enhancement on MR images were also demonstrated in most of the tumors, and cystic or hemorrhagic changes were noted in 2 cases.
\end{abstract}

S chwannoma is a benign slow-growing neoplasm composed entirely of nerve-supporting cells without neuronal elements. Although schwannomas are common in the head and neck, only $4 \%$ of schwannomas occur in the sinonasal cavity. ${ }^{1-3}$ Case series have generally described paranasal schwannoma as having nonspecific imaging findings, ${ }^{1,4}$ but there has been no large study describing the imaging features of sinonasal schwannoma, to our knowledge.

Complete surgical excision of a schwannoma is curative, and postoperative recurrence is rare. Therefore, correct preoperative diagnosis of sinonasal schwannoma is important because unnecessary extensive surgery can be avoided. ${ }^{5}$ A variety of benign or malignant tumors can occur in the sinonasal cavity, however, and their imaging features are too similar to allow accurate preoperative prediction of their histopathology. The purpose of this study was to report the characteristic CT and MR imaging findings of schwannomas in the sinonasal cavity.

\footnotetext{
Received April 13, 2012; accepted after revision June 13.

From the Departments of Otorhinolaryngology (Y.S.K., C.-H.K.) and Radiology (J.K.) Severance Hospital, Yonsei University College of Medicine, Seoul, Korea; and Department of Radiology (H.-J.K.), Samsung Medical Center, Sungkyunkwan University School of Medicine, Seoul, Korea.

Yoo Suk Kim and Hyung-Jin Kim contributed equally to this work.

Please address correspondence to Jinna Kim, MD, Department of Radiology, Severance Hospital, Yonsei University College of Medicine, 50 Yonsei-ro, Seodaemungu, Seoul 120-752, Korea; e-mail: jinna@yuhs.ac

http://dx.doi.org/10.3174/ajnr.A3257
}

\section{CASE SERIES}

\section{Patients}

Between January 2000 and July 2011, we encountered 12 patients with surgically proved sinonasal schwannoma at 2 large tertiary institutions. The patients included 7 men and 5 women, age range from 14 to 79 years, with a mean age of 37 years. We retrospectively reviewed CT $(n=12)$ and MR $(n=9)$ images of these 12 patients. Our institutional review board approved this retrospective study with a waiver of informed consent.

Nasal obstruction was the most common symptom, seen in 6 patients, followed by rhinorrhea $(n=2)$, anterior cheek pain $(n=$ $1)$, frequent epistaxis $(n=1)$, headache $(n=1)$, and exophthal$\operatorname{mos}(n=1)$. The duration of symptoms before diagnosis ranged from 1 to 48 months (mean, 10.4 months). None of the patients enrolled in the study had a history of previous sinonasal surgery.

\section{Imaging Analysis}

Among the 12 patients, 9 underwent both CT and MR imaging examinations, and 3 underwent only preoperative CT. CT scans were obtained by using a standard CT protocol for the paranasal sinuses. In 9 patients who had a lesion suspicious for tumor by endoscopic examination, precontrast and contrast-enhanced CT scans with a soft-tissue algorithm were obtained 40-60 seconds after intravenous administration of a nonionic contrast agent. In the remaining 3 patients, contiguous axial scans were acquired with a bone algorithm without the use of contrast agent. Precontrast T1-weighted spin-echo images and T2-weighted fast spinecho images with or without fat saturation were obtained, fol- 
Clinical and imaging findings in 12 patients with sinonasal schwannoma

\begin{tabular}{|c|c|c|c|c|c|c|c|c|c|c|c|}
\hline \multirow{2}{*}{$\begin{array}{l}\text { Patient/ } \\
\text { Age }(y r) / \\
\text { Sex }\end{array}$} & \multirow[b]{2}{*}{ Chief Symptom } & \multirow[b]{2}{*}{$\begin{array}{l}\text { CT/MR } \\
\text { Image }\end{array}$} & \multirow[b]{2}{*}{ Location } & \multirow[b]{2}{*}{$\begin{array}{l}\text { Size } \\
(\mathrm{mm})\end{array}$} & \multirow[b]{2}{*}{ Shape/Bone Erosion } & \multicolumn{2}{|r|}{ CT } & \multicolumn{3}{|c|}{ MR Imaging } & \multirow{2}{*}{$\begin{array}{l}\text { Cystic or } \\
\text { Hemorrhagic } \\
\text { Degeneration }\end{array}$} \\
\hline & & & & & & Attenuation $^{a}$ & Enhancement ${ }^{b}$ & $\begin{array}{l}\text { T1-Weighted } \\
\text { Image }^{\mathrm{a}}\end{array}$ & $\begin{array}{l}\text { T2-Weighted } \\
\text { Image }^{\mathrm{a}}\end{array}$ & Enhancement $^{\mathrm{b}}$ & \\
\hline $1 / 46 / \mathrm{F}$ & $\begin{array}{l}\text { Nasal obstruction for } \\
2 \mathrm{mo}\end{array}$ & Yes/yes & Lt. NC + ES & 52 & Tubular, expansile/yes & Isodense & Mild, patchy & Isointense & Isointense & Marked & Yes \\
\hline 2/27/M & $\begin{array}{l}\text { Anterior cheek pain for } \\
\quad 3 \mathrm{mo}\end{array}$ & Yes/yes & Lt. MS & 29 & Round, expansile/yes & Isodense & & Isointense & Isointense & Marked & No \\
\hline $3 / 24 / F$ & Exophthalmos for $1 \mathrm{mo}$ & Yes/yes & Lt. MS & 45 & Lobulated, expansile/yes & Isodense & Mild, patchy & Isointense & Isointense & Marked & Yes \\
\hline $4 / 14 / M$ & Rhinorrhea for $2 \mathrm{mo}$ & Yes/no & Septum & 14 & Round, nonexpansile/no & Isodense & Mild, patchy & & & & No \\
\hline $5 / 22 / M$ & $\begin{array}{l}\text { Nasal obstruction for } \\
\quad 6 \mathrm{mo}\end{array}$ & Yes/yes & Lt. NC & 55 & Tubular, expansile/yes & Isodense & Mild, patchy & Hypointense & Hyperintense & Marked & No \\
\hline $6 / 51 / M$ & $\begin{array}{l}\text { Nasal obstruction for } \\
\quad 9 \mathrm{mo}\end{array}$ & Yes/no & Lt. NC & 50 & Tubular, nonexpansile/no & Isodense & Mild, patchy & & & & No \\
\hline $7 / 42 / F$ & $\begin{array}{l}\text { Nasal obstruction for } \\
\quad 6 \mathrm{mo}\end{array}$ & Yes/yes & Rt. NC + ES & 45 & Lobulated, expansile/yes & Isodense & Marked & Hypointense & Isointense & Marked & No \\
\hline $8 / 33 / \mathrm{M}$ & $\begin{array}{l}\text { Nasal obstruction for } \\
\quad 4 \mathrm{mo}\end{array}$ & Yes/yes & Rt. NC + ES & 30 & Tubular, nonexpansile/no & Isodense & Mild & Isointense & Isointense & Marked & No \\
\hline $9 / 33 / F$ & $\begin{array}{l}\text { Nasal obstruction for } \\
3 \mathrm{mo}\end{array}$ & Yes/yes & Rt. NC + ES & 35 & Tubular, expansile/yes & Isodense & Mild & Isointense & Isointense & Marked & No \\
\hline $10 / 79 / \mathrm{M}$ & Rhinorrhea for $2 \mathrm{yr}$ & Yes/yes & Lt. septum & 15 & Round, nonexpansile/no & Isodense & Mild, patchy & Isointense & Isointense & Marked & No \\
\hline $11 / 45 / M$ & Epistaxis for $2 \mathrm{mo}$ & Yes/no & Rt. septum & 5 & Round, nonexpansile/no & Isodense & & & & & No \\
\hline $12 / 30 / \mathrm{F}$ & Headache for $1 \mathrm{mo}$ & Yes/yes & Lt. NC + ES & 45 & Lobulated, expansile/yes & Isodense & & Isointense & Isointense & Marked & No \\
\hline
\end{tabular}

Note:-ES indicates ethmoid sinus; NC, nasal cavity; MS, maxillary sinus; Rt., right; Lt., left.

${ }^{a}$ Compared with the attenuation and signal intensity of the brain stem.

${ }^{\mathrm{b}}$ Compared with the enhancement of the masseter muscle.
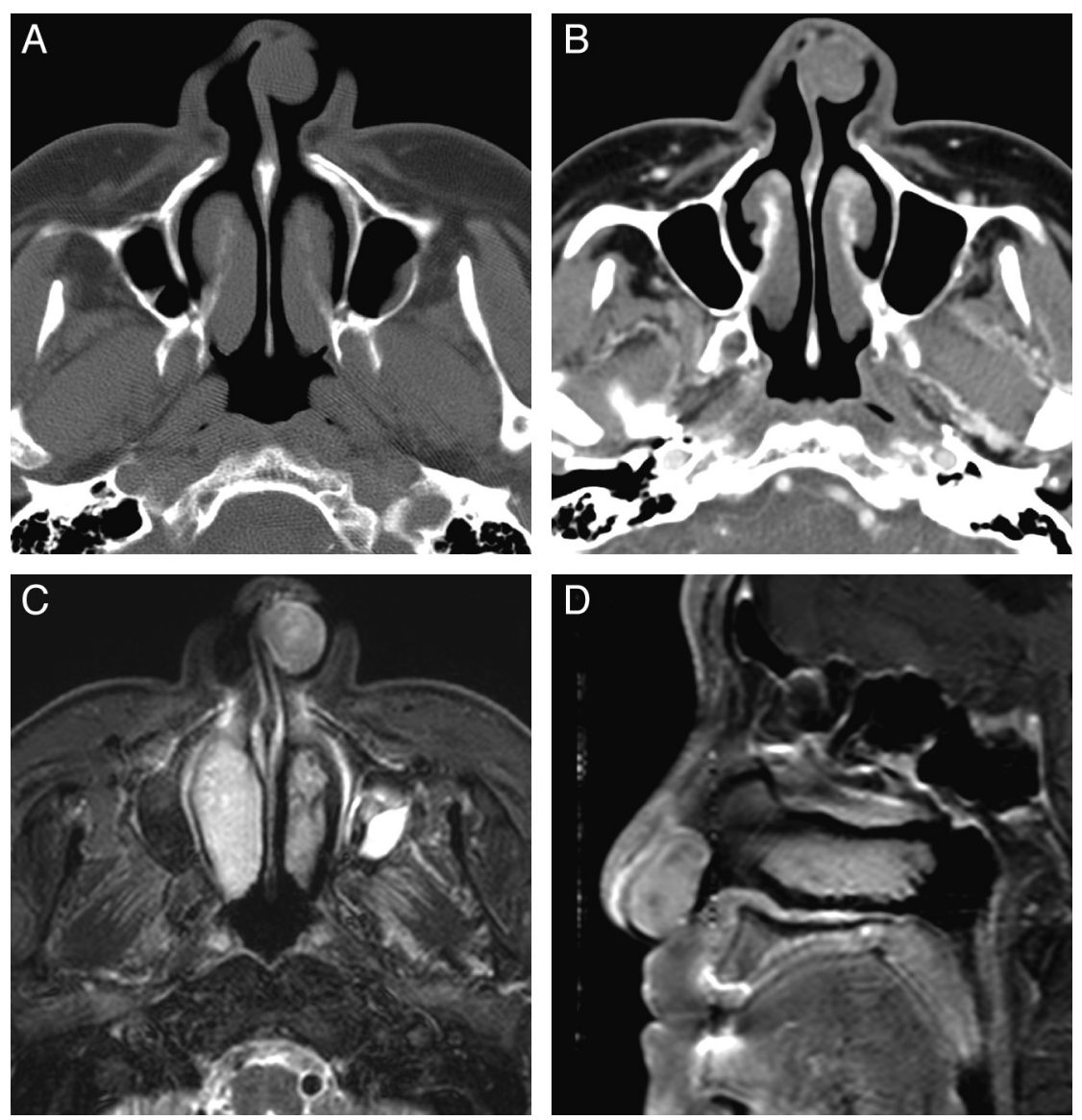

FIG 1. Case 10. Schwannoma of the nasal cavity in a 79-year-old man. A, Precontrast axial CT scan with a bone algorithm shows a polypoid mass in the left anterior nasal cavity, originating from the nasal septum. $B$, Contrast-enhanced axial CT scan with a soft-tissue algorithm shows mild and patchy enhancement of the mass. $C$, Fat-suppressed axial T2-weighted MR image shows that the mass is isointense to the brain stem. $D$, Contrast-enhanced fat-suppressed sagittal T1-weighted MR image shows marked contrast enhancement within the mass.

lowed by contrast-enhanced T1-weighted spin-echo images with $(n=7)$ or without fat saturation $(n=2)$.

Two experienced head and neck radiologists (with clinical experience of 22 years and 10 years) retrospectively reviewed all the
CT and MR images in consensus. The CT and MR imaging characteristics were analyzed with particular attention to the location, margin, shape, size, internal architecture, pattern and degree of enhancement of the tumor, and changes of the bony walls of the sinonasal cavity. The margin of the tumor was classified as well-defined or ill-defined. The size of each tumor was measured at the greatest diameter of the tumor. The internal architecture of each tumor was determined by attenuation on precontrast CT scans and signal intensity on T1- and T2-weighted MR images, which were compared with those of the brain stem. The presence of calcification and cystic or hemorrhagic change within the tumor, determined on precontrast CT scans and T1- and T2weighted MR images, respectively, was also recorded. On postcontrast CT and MR images, the degree of enhancement was also subjectively assessed as mild (enhancement less than or equal to that of the masseter muscle) or marked (enhancement greater than that of the masseter muscle).

\section{Imaging Findings}

The CT and MR imaging features of the 12 patients with sinonasal schwannoma are summarized in the Table. The tumors were seen as well-defined round $(n=4)$ (Fig 1$)$, tubular $(n=5)$ (Fig 2$)$, or partially lobulated soft-tissue masses $(n=3)$ (Figs 3 and 4$)$ in various locations in the sinonasal cavity, ranging from 0.5 to $5.5 \mathrm{~cm}$ (mean, $3.5 \mathrm{~cm}$ ). Ten tumors primarily involved the nasal cavity and/or ethmoid sinus, and the remaining 2 tumors involved the 

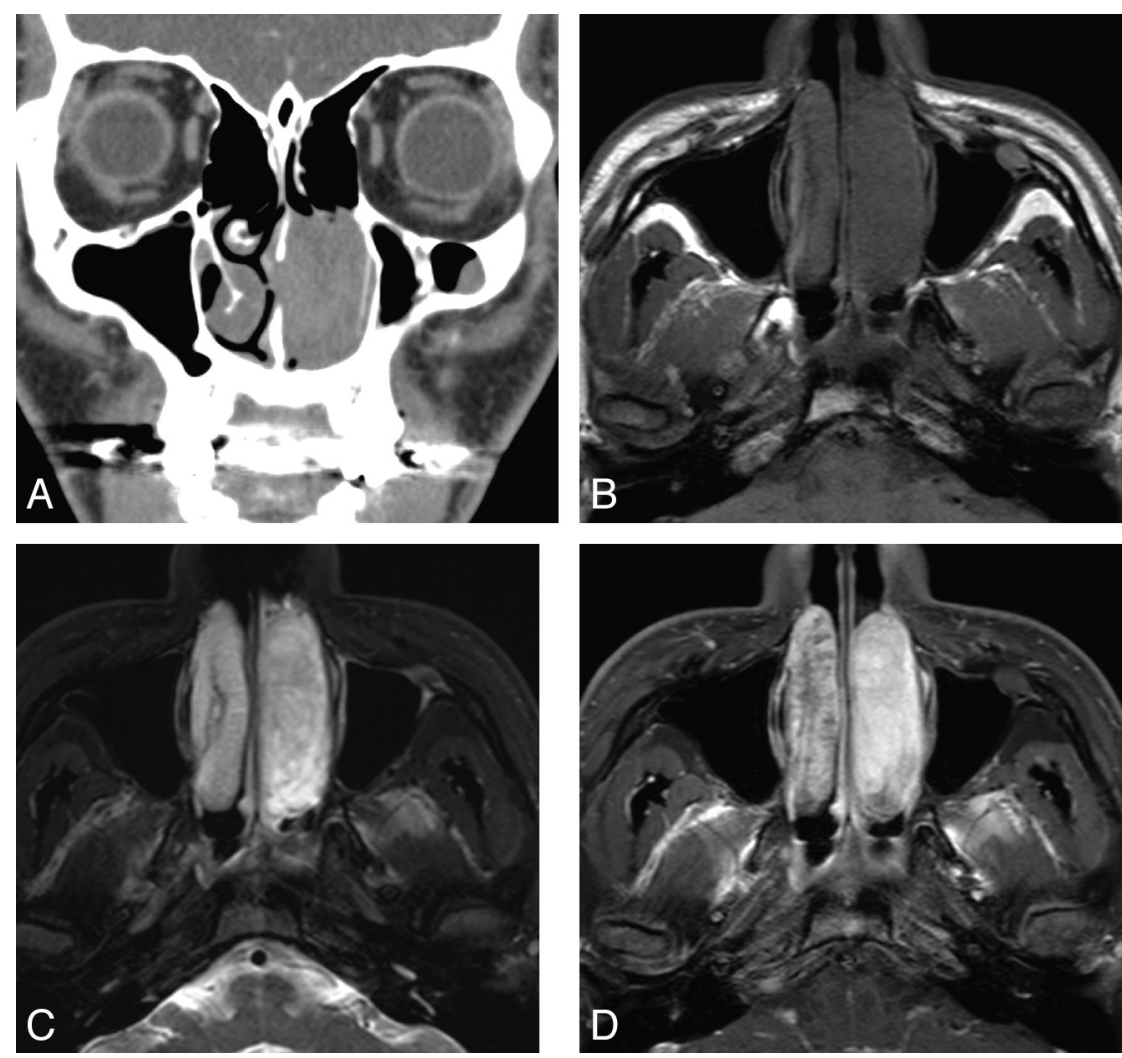

FIG 2. Case 5. Schwannoma of the nasal cavity in a 22-year-old man. A, Contrast-enhanced coronal CT image shows a tubular expansile soft-tissue mass in the left nasal cavity, demonstrating mild enhancement. $B$, Axial Tl-weighted MR image shows the tumor extending in the anteroposterior dimension and remodeling the lateral nasal wall. C, Fat-suppressed axial T2-weighted MR image shows that the tumor is hyperintense to the brain stem. $D$, Contrast-enhanced fat-suppressed axial T1-weighted MR images show marked homogeneous enhancement within the tumor.

maxillary sinus. The right side was involved in 4 tumors; the left side, in 7; and the midline, in 1 . Of the 10 tumors that primarily involved the nasal cavity and/or ethmoid sinus, 5 were located in the nasal cavity without any extension to the paranasal sinuses (Figs 1 and 2), 3 of which were confined to the nasal septum without contact with the lateral wall of the nasal cavity. The other 5 tumors simultaneously involved the nasal cavity and ethmoid sinus (Fig 3), with 3 centered in the posterior ethmoid sinus and 2 in the anterior ethmoid sinus, respectively. The remaining 2 tumors involving the maxillary sinus were associated with widening of the infraorbital canal (Fig 4). Seven tumors had the appearance of an expansile mass with pressure remodeling of the adjacent bony walls (Figs 3 and 4). One of these 7 tumors was a large mass in the right nasal cavity and posterior ethmoid sinus, extending into the ipsilateral orbit, maxillary sinus, sphenoid sinus, and the contralateral nasal cavity and ethmoid sinus with marked pressure remodeling of the adjacent bones (Fig 3). However, no aggressive destruction of surrounding bony structures, intracranial extension, or direct infiltration to the adjacent structures, as is often seen with a malignant tumor, was noted.

Compared with that of the brain stem, the attenuation of tumors seen on precontrast CT scans was isoattenuated in all cases. No case showed intratumoral calcification on precontrast CT scans. On the other hand, compared with that of the brain stem, 9 tumors examined by MR imaging showed isointensity $(n=7)$ or hypointensity $(n=2)$ on T1-weighted images and isointensity $(n=8)$ (Figs $1 C$ and 3C) or hyperintensity $(n=1)$ (Fig $2 C)$ on T2-weighted images. Contrast-enhanced CT images demonstrated mild $(n=8)$ (Figs $1 B$ and $2 A)$ or marked $(n=1)$ enhancement (Fig $3 B$ ), and relatively homogeneous marked enhancement was observed in all 9 tumors examined by contrast-enhanced MR imaging (Figs $1 D, 2 D, 3 D$, and $4 D$ ). Cystic changes were noted in 2 tumors, 1 of which also demonstrated intratumoral hemorrhage on MR images (Fig 4).

\section{DISCUSSION}

In our study, sinonasal schwannoma more frequently involved the nasal cavity and ethmoid sinus $(n=10)$ than other paranasal sinuses $(n=2)$; this location is in agreement with findings from previous reports. ${ }^{2,4,6,7}$ Sinonasal schwannomas are postulated to arise from the ophthalmic and maxillary branches of the trigeminal nerve or from autonomic nerves to the septal vessels and mucosa. ${ }^{6,7}$ In particular, the nasociliary and anterior ethmoid nerves (branches of ophthalmic nerve) innervate the anterior part of the nasal septum and anterior half of the lateral nasal wall, and the nasopalatine nerve (a branch of maxillary nerve) is responsible for the posterior and inferior areas of the nasal septum. All of the turbinate structures of the lateral nasal wall, however, are innervated by the posterior superior and inferior lateral nasal branches of the maxillary nerve. ${ }^{8}$

We hypothesized that schwannomas occur more frequently in the nasal cavity and ethmoid sinus than in other paranasal sinuses 

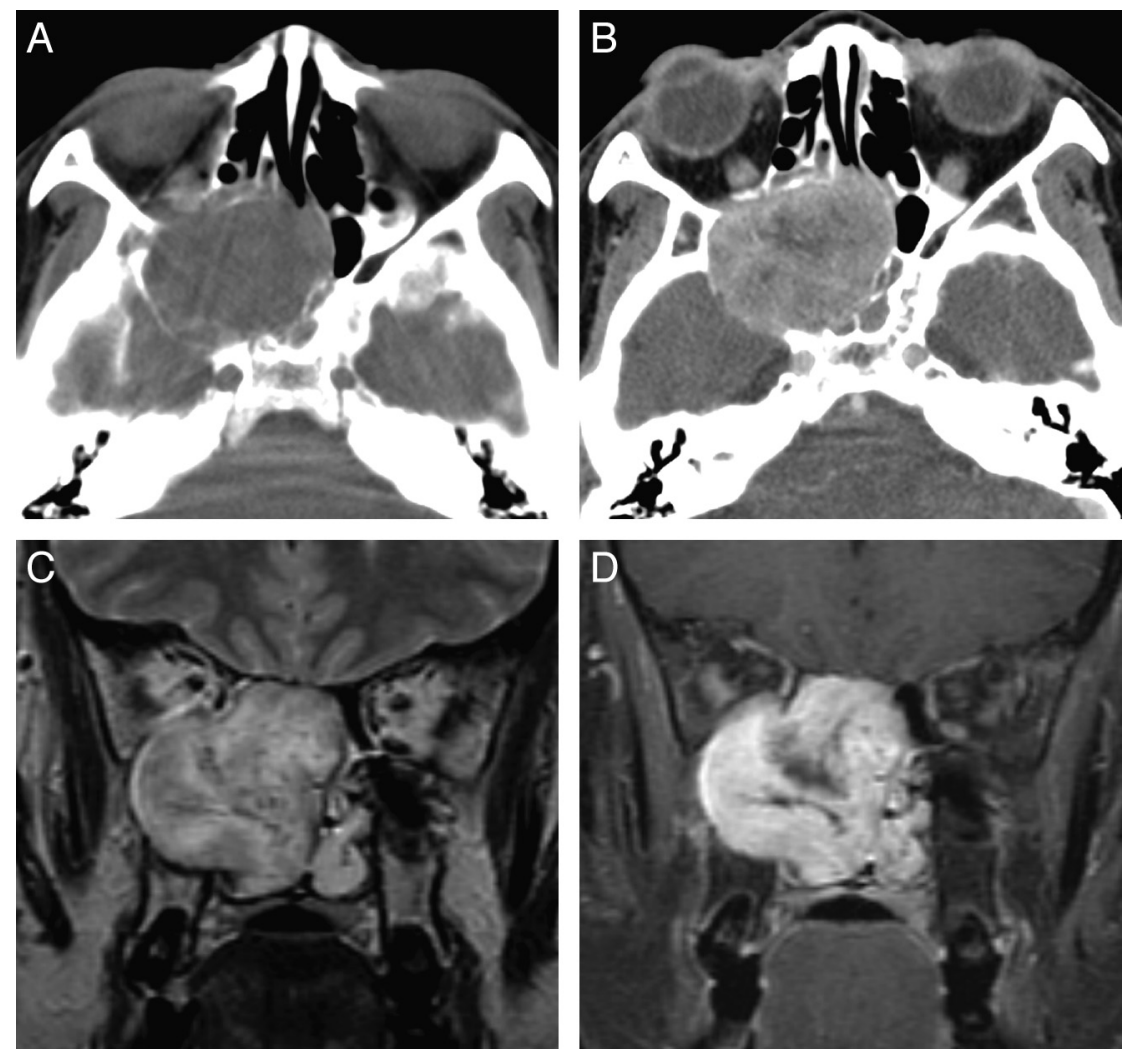

FIG 3. Case 7. Schwannoma of the nasal cavity and ethmoid sinus in a 42-year-old woman. A, Precontrast axial CT scan shows a large lobulated expansile mass isoattenuating to the brain stem and centered in the right posterior ethmoid sinus. B, Postcontrast axial CT scan shows marked enhancement of the mass, greater than that of the muscles in the masticator space. The mass extends to the ipsilateral orbit and maxillary and sphenoid sinuses with scalloping and remodeling of the bony walls of the nasal septum, maxilla, and sphenoid bone. C, Coronal T2-weighted MR image shows heterogeneous signal intensity of the mass and signal voids within the lesion, suggestive of prominent vascularity. $D$, Contrastenhanced fat-suppressed coronal T1-weighted MR image shows marked and heterogeneous enhancement of the mass.

because of their more abundant and complex nerve innervations. Another possible explanation is that schwannomas of the nasal cavity may cause earlier and more frequent symptoms, such as nasal obstruction and epistaxis, than lesions in other paranasal sinus locations, which enhances detection of these tumors. In many cases, the nerve of origin cannot be precisely identified during surgery and most schwannomas do not present with referable neurologic symptoms; therefore, it is difficult to know the exact incidence of schwannoma according to nerve origin in the sinonasal cavity.

In the present study, 7 of the 12 tumors (58.3\%) were expansile with smooth erosion and scalloping of the adjacent bony walls (Figs 2-4); none had invasive features seen in malignancy. Schwannomas arising from the nasal septum were small and round, but most tumors arising from the nasal cavity and ethmoid sinus had a relatively characteristic tubular shape along the anteroposterior or superoinferior axis of the nasal cavity (Fig 2), which presumably resulted from the soft nature and slow growth pattern of the schwannoma, without destruction and invasion of adjacent anatomic structures. The lamellae, which are composed of thin bone constituting the ethmoid air cells, are L-shaped structures. Just as the lower horizontal portion of the lamellae unite with the inferior part of the anterior vertical portion of the lamellae and are attached to the skull base, schwannomas of the nasal cavity might grow according to the alignment of the ethmoid lamellae and result in a tubular shape, extending anteroposteri- orly or superoinferiorly. Therefore, we hypothesize that tubular tumor shape is a helpful clue for differentiating schwannoma in the nasal cavity from more common and more invasive sinonasal malignancies.

Schwannomas arising from other parts of the body display relatively consistent imaging features, and most schwannomas show intermediate $\mathrm{T} 1$ and variable $\mathrm{T} 2$ signal intensity on MR imaging. ${ }^{9-12}$ In this study, we thought that it was not adequate to select skeletal muscle as an object for the comparison of MR signal intensity because almost all schwannomas show higher T2 signal intensity than skeletal muscle. Therefore, we compared the signal intensity of the solid part of the schwannoma with that of the brain stem, and most of the sinonasal schwannomas (8 of 9 , $88.9 \%$ ) were isointense with the brain stem on T2-weighted MR images in our study (Figs $1 C$ and $3 C$ ). This might reflect the fact that schwannomas arising from the sinonasal cavity are prone to be more cellular, mostly composed of Antoni A areas, rather than cystic or stromal and Antoni B areas, and this feature might, in turn, result in the lower T2 signal intensity of sinonasal schwannoma than schwannomas in other parts of the body.

Hasegawa et $\mathrm{al}^{13}$ and Sheikh et $\mathrm{al}^{14}$ each reported a series of 5 cases of sinonasal schwannoma; and 4 and 3 cases, respectively, were the cellular variant composed of a compact proliferation of spindle-shaped cells arranged in interlacing fascicles with predominantly hypercellular Antoni A areas on histopathologic examination. These pathologic reports also support our suggestion 

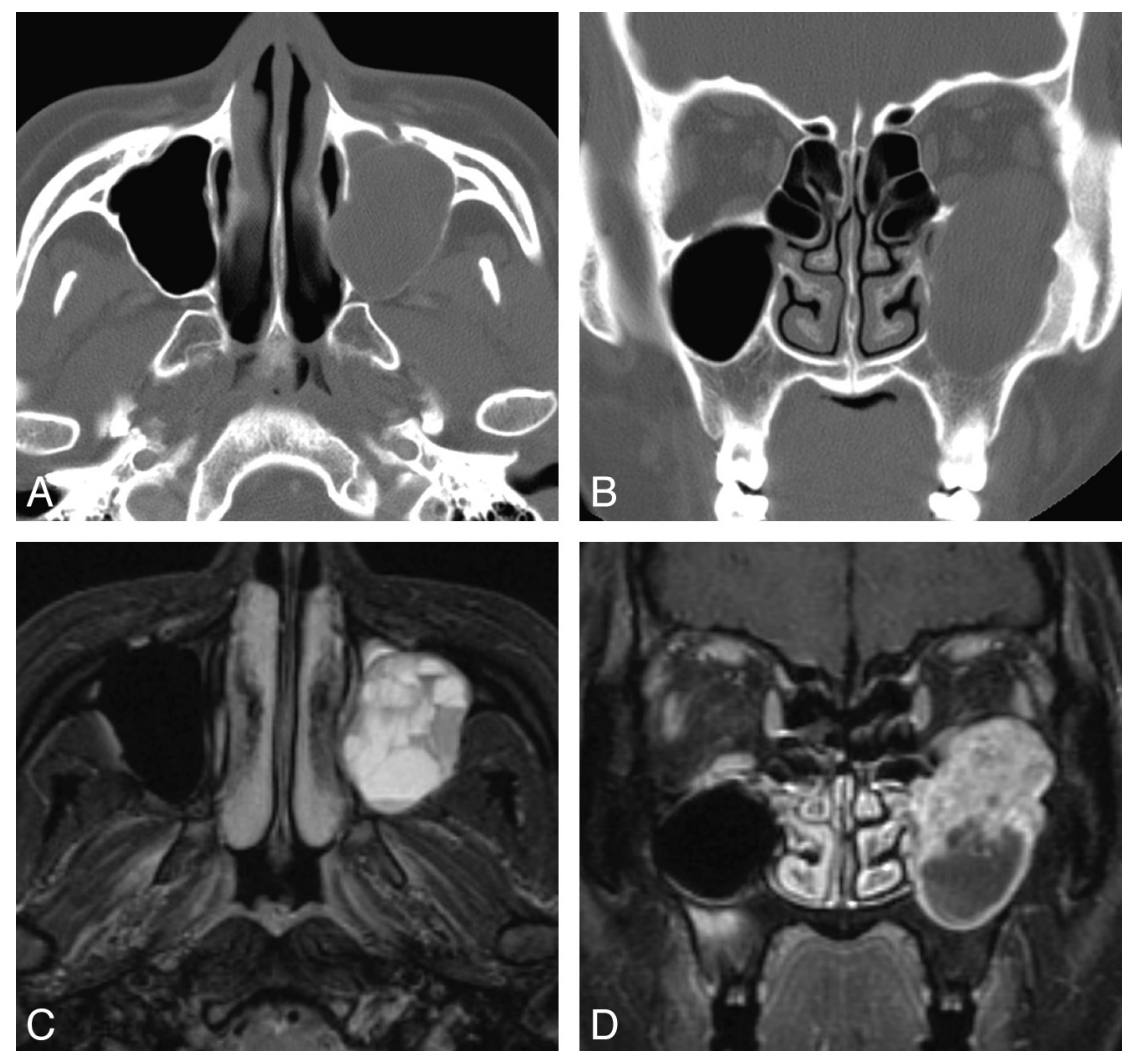

FIG 4. Case 3. Schwannoma of the maxillary sinus in a 24-year-old woman. $A$ and $B$, Precontrast axial and coronal CT scan with a bone algorithm shows a lobulated expansile mass arising from the left infraorbital canal, which replaces the left maxillary sinus. Note cortical thinning and remodeling of the orbital floor and the medial and posterior maxillary sinus walls by the mass. C, Fat-suppressed axial T2-weighted MR image shows multiple fluid-fluid levels within the lesion, which are suggestive of intratumoral hemorrhage. $D$, Contrast-enhanced fat-suppressed coronal T1-weighted MR image shows cystic change at the lower part of the mass and marked enhancement in the upper solid part of the mass.

that sinonasal schwannomas tend to show lower T2 signal intensity than schwannomas in other body parts. In addition, their T2 signal characteristics might make differentiation from other sinonasal tumors more difficult on MR imaging because virtually all sinonasal tumors are highly cellular with an intermediate signal intensity on T2-weighted MR images.

As expected, MR imaging was more sensitive to the presence of contrast enhancement than CT. While most of the schwannomas ( 8 of $9,88.9 \%$ ) showed a mild and patchy enhancement pattern on contrast-enhanced CT, all tumors showed marked enhancement on contrast-enhanced MR imaging. Schwannomas are hypovascular tumors but can show marked and delayed enhancement on images acquired beyond 60 seconds after the contrast injection due to pooling of contrast from poor venous drainage. ${ }^{2}$ Therefore, we hypothesize that the different degree of enhancement of schwannomas on CT and MR imaging can provide useful information for suggesting a diagnosis of sinonasal schwannoma preoperatively.

Most interesting, 1 case showed conspicuously strong enhancement that was greater than skeletal muscle on CT (Fig 3B), though the interval between image acquisition and contrast injection was not exactly the same in our study (40-60 seconds after contrast administration). A few studies have reported unusually early and strong enhancement of schwannomas on CT, showing signs of prominent vascularity within the tumor or around the capsule, but the exact incidence and histopathologic differences from hypovascular schwannoma have not been described. ${ }^{15,16}$ Therefore, some schwannomas can show unusual hypervascularity on CT, and this is a potential diagnostic pitfall.

Cystic or hemorrhagic degeneration of schwannomas is a characteristic feature, especially in large tumors, ${ }^{9-11}$ and MR imaging better demonstrates the signal intensities of cystic or hemorrhagic degeneration than CT due to higher soft-tissue contrast resolution. In our study, 2 patients (16.7\%) showed marked heterogeneity and obvious areas of cystic or hemorrhagic degeneration (Fig 4). We hypothesize that the relative scarcity of degenerative changes of nasal cavity schwannomas in our study might be associated with the smaller size and earlier detection due to clinical symptoms compared with schwannomas in other body parts.

In addition, although schwannomas have a true capsule composed of dense and thick fibrotic tissue surrounding the tumor parenchyma, exact 1:1 imaging-pathologic correlation could not be performed in our study because most of the schwannomas were removed in pieces and not en bloc. ${ }^{12,17}$ We delineated the tumor capsule on pathologic examination in 2 cases after surgery (patients 1 and 3), but it was difficult to identify a fibrous capsule on MR imaging because of confusion with the low signal intensity of adjacent compressed tissue such as the bony lamellae of the ethmoid sinus. Better imaging delineation of the fibrous capsule surrounding schwannomas would be helpful in differentiating schwannomas from other sinonasal neoplasms, but the sinonasal 
cavity has anatomic features (air, bone) mentioned above that make identification of the capsule difficult on imaging.

\section{CONCLUSIONS}

Sinonasal schwannoma is generally a well-defined soft-tissue mass most frequently occurring in the nasal cavity and ethmoid sinus and frequently associated with pressure remodeling of the adjacent bone. These tumors are isoattenuating on CT and predominantly isointense on both T1- and T2-weighted MR images, compared with the brain stem. Mild contrast enhancement on CT and strong enhancement on MR images were also demonstrated in most of the tumors. Although sinonasal schwannomas are rare and their imaging findings are rather nonspecific, CT and MR imaging studies are helpful for preoperative diagnosis and surgical planning in patients with schwannoma of the sinonasal cavity.

\section{REFERENCES}

1. Yu E, Mikulis D, Nag S. CT and MR imaging findings in sinonasal schwannoma. AJNR Am J Neuroradiol 2006;27:929-30

2. Som PM, Biller HF, Lawson W, et al. Parapharyngeal space masses: an updated protocol based upon 104 cases. Radiology 1984;153: $149-56$

3. Hillstrom RP, Zarbo RJ, Jacobs JR. Nerve sheath tumors of the paranasal sinuses: electron microscopy and histopathologic diagnosis. Otolaryngol Head Neck Surg 1990;102:257-63

4. Dublin $\mathrm{AB}$, Dedo $\mathrm{HH}$, Bridger WH. Intranasal schwannoma: magnetic resonance and computed tomography appearance. Am J Otolaryngol 1995;16:251-54

5. Pasquini E, Sciarretta V, Farneti G, et al. Endoscopic endonasal ap- proach for the treatment of benign schwannoma of the sinonasal tract and pterygopalatine fossa. Am J Rhinol 2002;16:113-18

6. Hegazy HM, Snyderman CH, Fan CY, et al. Neurilemmomas of the paranasal sinuses. Am J Otolaryngol 2001;22:215-18

7. Buob D, Wacrenier A, Chevalier D, et al. Schwannoma of the sinonasal tract: a clinicopathologic and immunohistochemical study of 5 cases. Arch Pathol Lab Med 2003;127:1196-99

8. Levin HL, Clemente MP. Sinus Surgery: Endoscopic and Microscopic Approaches. New York: Thieme Medical Publisher; 2004:24-29

9. Cohen LM, Schwartz AM, Rockoff SD. Benign schwannomas: pathologic basis for CT inhomogeneities. AJR Am J Roentgenol 1986; 147:141-43

10. Beaman FD, Kransdorf MJ, Menke DM. Schwannoma: radiologicpathologic correlation. Radiographics 2004;24:1477-81

11. Rha SE, Byun JY, Jung SE, et al. Neurogenic tumors in the abdomen: tumor types and imaging characteristics. Radiographics 2003;23: $29-43$

12. Anil G, Tan TY. Imaging characteristics of schwannoma of the cervical sympathetic chain: a review of 12 cases. AJNR Am J Neuroradiol 2010;31:1408-12

13. Hasegawa SL, Mentzel T, Fletcher CD. Schwannomas of the sinonasal tract and nasopharynx. Mod Pathol 1997;10:777-84

14. Sheikh HY, Chakravarthy RP, Slevin NJ, et al. Benign schwannoma in paranasal sinuses: a clinico-pathological study of five cases, emphasising diagnostic difficulties. J Laryngol Otol 2008;122:598-602

15. Aygenc E, Selcuk A, Ozdem C. Hypervascular parapharyngeal schwannoma: an unusual case. Auris Nasus Larynx 2002;29:215-17

16. Quiroga S, Alvarez-Castells A, Pallisa E, et al. Duodenal schwannoma causing gastrointestinal bleeding: helical CT findings. Abdom Imaging 1997;22:154-55

17. Sharma R, Tyagi I, Banerjee D, et al. Nasoethmoid schwannoma with intracranial extension. Case report and review of literature. Neurosurg Rev 1998;21:58-61 